\section{Privileged beginnings}

Under certain conditions, foreign haematopoietic stem cells can engraft in a new host, giving rise to a chimaeric immune system. One benefit of this is that the donorderived immune cells induce tolerance to the donor major histocompatibility complex (MHC) antigens. This tolerance allows acceptance of organ and tissue grafts from the same donor. However, contaminating $\mathrm{T}$ cells in the transfusion can respond to the recipient's MHC antigens and cause a lethal graft-versus-host reaction. Also, allogeneic haematopoietic stem cells are usually rejected outright unless the recipient's immune system is depleted and suppressed ('host conditioning'). Embryonic stem (ES) cell transfusions, however, carry no risk of contaminating $\mathrm{T}$ cells and, as Fred Fändrich and colleagues show in the February issue of Nature Medicine, they have a crucial immunological advantage over haematopoietic stem cells.

An implanted embryo is, in essence, an allograft: half of the MHC antigens expressed are paternal. Studies indicate that the implantation-stage embryo from which ES cells can be derived - has intrinsic mechanisms of avoiding rejection by the maternal immune system. This new study investigated whether ES cells also have a special immune-privilege status that might allow them to engraft in an allogeneic host.

Stable ES-cell lines were derived from rat blastocysts. These stem cells express few or no MHC molecules. The stem cells were then labelled with a fluorescent dye and transferred by intravenous injection into fully MHC-disparate rats. Seven days after injection, fluorescent cells were detected in the liver, spleen, thymus and mesenteric lymph nodes. A proportion of these cells had become positive for MHCclass-II molecules, indicating that differentiation had commenced. In the blood, staining for a donor MHC-class-I molecule revealed that $5-8 \%$ of leukocytes were now derived from the implanted stem cells. This proportion stabilized at $6-12 \%$. The leukocytes of donor origin included B cells and monocytes but, strikingly, no $\mathrm{T}$ or NK cells.

What mechanism might account for the survival of embryonic stem cells in allogeneic hosts in the absence of host conditioning? Expression of the death receptor ligand Fas ligand (FasL), seems to be high on the rat ES cells. Also, the rat ES cells were shown to trigger Fas-dependent killing of activated $\mathrm{T}$ cells in vitro. This indicates that there is a role for FasL, but other tolerance mechanisms also need to be investigated.

So, does the stable chimaerism permit acceptance of an organ transplant? To test this, heart allografts of the same genotype as the ES cells were transplanted into the abdominal cavity of the chimaeric rats. As expected, the transplants were permanently accepted.

This study has important implications for both transplantation and stem-cell therapies in general. If resistance to immune rejection is unique to specialized ES cells then they might become the stem cell of choice for therapeutic purposes. In organ transplantation, ES-cellinduced tolerance could eliminate the requirement for immunosuppression and tissue matching of recipient and organ donor. However, organ and stem cell would of course have to be MHC matched. This would require a large bank of human ES-cell lines of known MHC haplotype, which raises substantial logistical and ethical concerns.

Jennifer Bell

(4) References and links ORIGINAL RESEARCH PAPER Fändrich, $F$ et al. Preimplantation-stage stem cells induce long-term allogeneic graft acceptance without supplementary host conditioning. Nature Med. 8, 171-178 (2001).

FURTHER READING Nature Insight: Stem cells. Nature 414, 88-131 (2001).

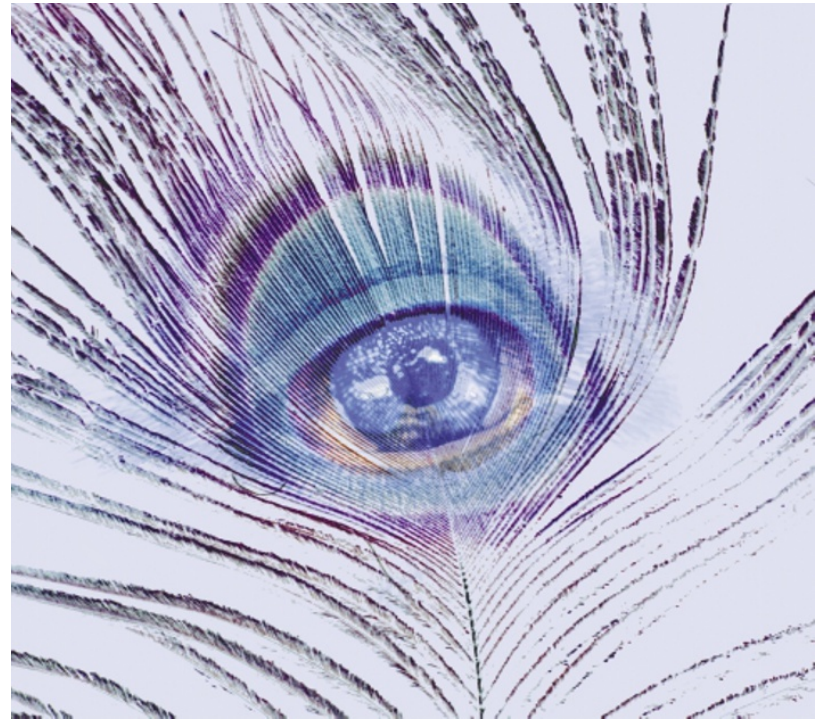

LYMPHOCYTE DEVELOPMENT

\section{Appearances can be deceptive}

Gene-targeting studies have been invaluable for dissecting the role of specific genes in lymphocyte development. B and T cells, as well as natural killer (NK) cells and some dendritic cells, develop in the thymus from multipotent lymphoid progenitors (MLPs). The signalling pathways that influence the development of MLPs and their progeny are not well understood. Mice that do not express the receptor for interleukin 7 (IL-7) (IL-7R $\alpha$ ) or the transcription factor E47 (encoded by the E2A gene) have very similar defects in B- and T-cell development, which indicates that these proteins might function in the same signalling pathways. However, Kee and colleagues now report in EMBO Journal that IL-7-mediated signalling and E47 function independently to promote the development of MLPs.

IL-7R signalling is required for expression of the antiapoptotic protein $\mathrm{BCL} 2$, and the defects in $\mathrm{T}$ - and B-cell development and survival in $\mathrm{Il}-7 \mathrm{rO} \mathrm{K}^{-/}$mice are rescued when these mice are crossed with $B c l 2$ transgenic mice. By contrast, the authors show that expression of the $\mathrm{Bcl} 2$ transgene in the $E 47^{-/-}$mice does not rescue the lymphopoietic defects in these animals. In addition, $\mathrm{CD} 25^{+} \mathrm{CD} 4^{-} \mathrm{CD} 8^{-}$ $E 47^{--}$thymocytes are highly sensitive to apoptosis in vitro and more cells express the proapoptotic protein caspase-3. These results indicate that E2A proteins promote lymphocyte survival through a BCL2-independent pathway.

Considering that BCL2 is one of the main targets of the IL-7 survival pathway, does this mean that E2A proteins function independently of IL-7 signalling? To address this, Kee and colleagues generated $\mathrm{Il}-7 \mathrm{r} \mathrm{\alpha}^{-/-} \times E 47^{-/-}$double knockout mice. All subsets of thymocytes were severely reduced in the double knockout mice and thymocyte development was blocked before T-cell commitment. Thymic NK cells were also reduced. Therefore, despite similarities between the IL-7R $\alpha$ and E47 single knockouts, the more severe phenotype seen in the double knockouts indicates that IL-7R $\alpha$ and E47 act in distinct pathways to promote the survival of MLPs.

Jenny Buckland

(Q) References and links ORIGINAL RESEARCH PAPER Kee, B. et al.

IL-7R $\alpha$ and E47: independent pathways required for development of mulitpotent lymphoid progenitorsitle. EMBO J. 21, 103-113 (2002).

FURTHER READING Engel, I. \& Murre, C. The function of E- and ld proteins in lymphocyte development. Nature Rev. Immunol. 1, 193-199 (2001).

\section{WEB SITE}

Cornelis Murre's laboratory: http://www.biology.

ucsd.edu/labs/murre/ 